\title{
Biologically Relevant Universality of Move F in Wh-Questions
}

\author{
Daoshan Ma \\ School of Foreign Languages, Tianjin Polytechnic University, Tianjin, China \\ Email:madaos@tom.com
}

How to cite this paper: Ma, D.S. (2018) Biologically Relevant Universality of Move F in Wh-Questions. Open Access Library Journal, 5: e4442.

https://doi.org/10.4236/oalib.1104442

Received: February 23, 2018

Accepted: March 12, 2018

Published: March 15, 2018

Copyright ( 2018 by authors and Open Access Library Inc.

This work is licensed under the Creative Commons Attribution International License (CC BY 4.0).

http://creativecommons.org/licenses/by/4.0/

\begin{abstract}
The thesis deals with the four types of languages from the perspective of wh-questions which prove to exhibit a universal principle of Move $\mathrm{F}$ and investigates on the biologically basis of language studies. The feature checking of the wh-questions in these four types of languages can be thus given a principled explanation and Move $\mathrm{F}$ as a universal principle is biologically related.
\end{abstract}

\section{Subject Areas}

Education, Linguistics

Keywords

Biolinguistics, Universal Principle, Feature Checking, Move F

\section{Introduction}

Biolinguistics regards the study of language faculty as an essential part of biology. The biological basis of language studies deals with "which apparent principles of language ... are unique to this cognitive system" and "how much of language can be given a principled explanation" [1]. The wh-questions in the world exhibit four types of wh-movement and from the important divergences of the four types of languages, a universal principle of Move F can be derived [2] [3]-[9]. If "things mental" can be produced by principles, what "things mental" can Move F generate? Is Move F a common principle related with some biological factors of the mind behind these apparent overt different properties of languages? If it is, the nature and nurture problem may be answered by the biologically relevant universality. As it is proven that "things mental ... are emergent properties of the brains ... produced by principles" [1]. It is thus believed that a person's language is "a state of some component of the mind" [1]. 
Natural languages can be classified into four types from the perspective of wh-movement in wh-questions [2]-[9]. The first type of languages is English, in which only one wh-word is required to be raised to the spec position of $\mathrm{CP}$ in the sentence initial position of the wh-questions, which is referred to as a Single filled spec language. The second type of languages is Chinese [10] [11] [12], in which no wh-word is triggered to move to the spec position of CP in the sentence initial position of the wh-questions [13]-[17], which is called a null spec language. The third type of languages is multiply filled spec language, in which the spec of $\mathrm{CP}$ is filled with more than one wh-word. The fourth type is the non-multiply filled spec language in which one wh-word is raised to the initial position in the spec CP and the other wh-words move to spec IP.

In the first type of languages, only one wh-word is triggered to move into the spec CP position of the wh-questions to check the strong [+wh] feature of the head C. In the second type of languages, no wh-word is needed to move to spec $\mathrm{CP}$ position as the [+wh] feature of the head $\mathrm{C}$ is said to be weak. One of the third type of languages is Bulgarian, in which all wh-words in the sentence must move to spec $\mathrm{CP}$ position required for feature checking, and all the wh-words form a mutual wh-operator and move together as a cluster to spec CP position for feature checking of the strong specifier feature of the head C. Czech is the fourth type of language in which only one wh-word can move to spec CP position and at most another wh-word can be adjoined to the moved wh-word in spec CP position and other wh-word or wh-words must move to spec IP position.

Is there a common principle that all these four types of natural languages follow in the varied kinds of wh-movement? The author argues that Move F is a universal principle that underlies the different wh-movements of the wh-questions in the natural languages. This universal principle is due to genetic factors rather than epigenetic or non-language specific factors. The important differences oriented on Move F are attributed to parameter selection or biological selection, if we may dare to say.

\section{Universality of Move F}

Chomsky says, "The minimal operation, then, should raise just the feature F" [18]. That's why we will propose that movement of the feature Move F should be a universal operation.

1) Move $F$

$$
K=\{\gamma,\{\alpha, \beta\}\}
$$

where $\alpha, \beta$ are features of syntactic objects already formed.

Chomsky [18:262] answers the reason why the [+wh] feature $\mathrm{F}$ does not raise alone to form $\{\gamma,\{\mathrm{F}, \mathrm{K}\}\}$ when $\mathrm{F}$ is moved to target $K$. Why isn't the formal feature of the wh-word involved in feature checking raised to the relevant spec CP position? The reason lies in the following economy principle.

2) Economy Principle 
F carries along just enough material for convergence.

3) Which book did you read?

In the English sentence 3), an extra element is required to move along with the wh-feature for convergence which involves a kind of generalized pied-piping, according to Chomsky. Generally speaking, bare output conditions might determine just what is carried along i.e. pied-piped when the feature $\mathrm{F}$ is moved. Chomsky assumes that the properties of phonological component require such pied-piping [18]. Therefore, in 3), the principle Move F pied-pipes the morphological feature of the wh-word "which" and leaving the residue "book" behind. This too crashes at PF. The wh-word "which" cannot be raised, because it is not a syntactic constituent subject to movement. In fact, the smallest element that can be moved as a category in the sentence of 3) is the DP "which book". According to Chomsky, in light of the computational procedure, it is only the feature [+wh] $\mathrm{F}$ that is raised, the rest is carried along by virtue of the economy principle of 2). This also shows that Move $\mathrm{F}$ is a universal operation as it accords with economy principle while pied-piping of the extra element is conditional. As 3) illustrates, Move F in English automatically carries along FF (LI), the set of formal features of the lexical item. Thus Move F in English must follow 4), where FF (F) is FF (LI), F a feature of the lexical item LI, according to Chomsky.

4) Move F "carries along” FF (F).

In 3), the operation Move raises $\mathrm{F}$ and automatically raises the formal feature FF (F) too, carrying along the phrase "which book" containing F only when the movement of the feature $\mathrm{F}$ is overt, otherwise the derivation will crash. In the Chinese sentence 5), the operation Move raises $\mathrm{F}$ and does not automatically raise the formal feature FF (F), leaving the phrase "na ben shu"/"which book" containing $\mathrm{F}$ in situ because the movement is covert, and according to Chomsky, in covert movement, only features $\mathrm{F}$ raise alone. Thus the essence of Last Resort can also be kept as a property of the universal operation of Move F.

5) ni du le na ben shu?

You read past which cl. book

"Which book did you read?"

6) Last Resort as a property of the operation Move F.

$\mathrm{F}$ is unchecked and enters into a checking relation with a sublabel of $K$ as a result of the operation.

Chomsky [18] further develops the theory of the operation Move. Move raises the feature $\mathrm{F}$ to target $K$ in $\Sigma$ only if 7) holds, with 8 a) and 8 b) as automatic consequences and $8 \mathrm{c}$ ) a further consequence.

7) Last Resort

a) $\mathrm{F}$ is an unchecked feature.

b) $\mathrm{F}$ enters into a checking relation with a salable of $K$ as a result of the operation.

8) Move F

a) $\mathrm{FF}(\mathrm{F})$ raises along with $\mathrm{F}$.

b) A category $\alpha$ containing F moves along with F only as required for conver- 
gence.

c) Covert operations are pure feature raising.

According to the theory of Move mentioned by Chomsky in 7) and 8), in 3) the $[+w h]$ feature of the wh-phrase "which book" is an unchecked feature and thus this feature enters into a checking relation with a sublabel of $K$ as a result of the movement operation. The $[+w h]$ feature of the wh-phrase "which book" actually enters into a checking relation with the specifier [+wh] feature of the head $\mathrm{C}$ in $\mathrm{CP}$ position. In the checking process, Move $\mathrm{F}$ automatically carries along the FF (F) formal feature of the lexical item the wh-phrase "which book". The category DP "which book" containing F the [+wh] feature of the wh-phrase "which book" pied-pipes with F only as deeded for convergence. The category DP "which book" containing F the [+wh] feature of the wh-phrase "which book" pied-pipes with $\mathrm{F}$ into spec $\mathrm{CP}$ to check the specifier [+wh] feature of the head. And only when the category DP "which book" containing F the [+wh] feature of the wh-phrase "which book" is raised into spec CP, the specifier [+wh] feature of the head can be checked. The [+wh] feature of the category DP "which book" containing F the [+wh] feature of the wh-phrase "which book" agrees with the specifier [+wh] feature of the head and thus the specifier [+wh] feature of the head is checked. As the specifier [+wh] feature of the head is uninterpretable, once it is checked it is deleted and does not enter into LF. The derivation converges and the sentence is grammatical. In the Chinese sentence 5), however, only pure feature movement is required. In 5) as the specifier [+wh] feature of the head is weak, the FF (F) feature is not carried along with Move F. While in English the specifier [+wh] feature of the head is strong, the FF (F) features of the category DP "which book" must be carried along with Move F. There are four types of features as shown below [18]:

9) a) categorical features;

b) $\Phi$-features;

c) Case features;

d) Strong F, where F is categorical.

The structure of 3) can be illustrated by the representation of 10).

10) [ ${ }_{\mathrm{CP}}$ which book ${ }_{\mathrm{i}} \mathrm{I}_{\mathrm{IP}}$ did you read $\left.\mathrm{t}_{\mathrm{i}}\right]$ ].

The tree diagram of 3 ) is 11).

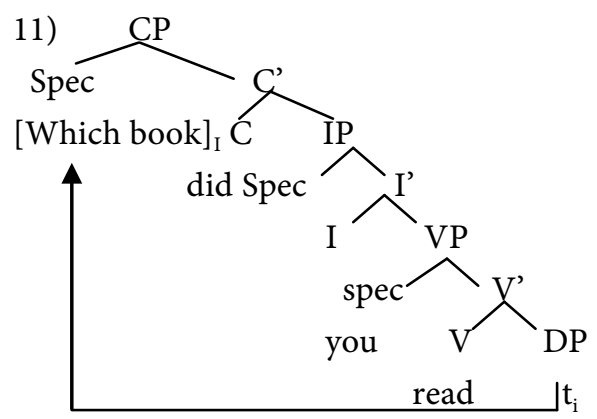

Similarly the structure of the English sentence 12) is 13), as is illustrated in the tree diagram 14).

12) Who read which book? 
13) $\left[\mathrm{CP}[+ \text { who }]_{\mathrm{i}}\left[\right.\right.$ IP $\mathrm{t}_{\mathrm{i}}$ read which book?]]

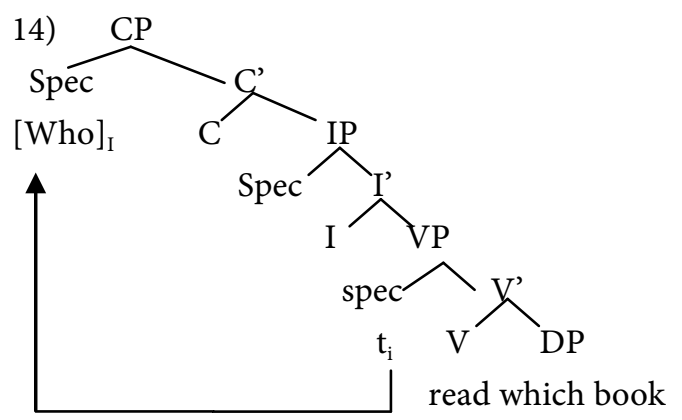

According to the theory of Move represented in 7) and 8), in 12) the [+wh] feature of the wh-word "who" is an unchecked feature and thus this feature enters into a checking relation with a sublabel of $K$ as a result of the movement operation. The [+wh] feature of the wh-word "who" actually enters into a checking relation with the specifier [+wh] feature of the head $\mathrm{C}$ in $\mathrm{CP}$ position. In the checking process, Move F automatically carries along the formal feature FF (F) of the lexical item of the wh-word "who". The category DP "who" containing F the [+wh] feature of the wh-word "who" moves along with F only as required for convergence. The category DP "who" containing F the [+wh] feature of the wh-word "who" moves along with F into spec $\mathrm{CP}$ to check the specifier [+wh] feature of the head C. And only when the category DP "who" containing F the [+wh] feature of the wh-word "who" moves into spec CP position, the specifier [+wh] feature of the head $\mathrm{C}$ can be checked. The [+wh] feature of the category DP "who" containing F the [+wh] feature of the wh-word "who" agrees with the specifier [+wh] feature of the head and thus the specifier [+wh] feature of the head is checked. And as the specifier [+wh] feature of the head is uninterpretable, once it is checked it is deleted and does not enter into LF. The derivation converges and the sentence is acceptable.

In 12) the wh-word "who" takes a wide scope over the lower wh-phrase "which book". As the wh-word "who" carries the interrogative [+wh] feature F, the [+wh] feature $\mathrm{F}$ is optimally raised to the spec $\mathrm{CP}$ position, and according to the economy principle in 2) and move operation in 4), this movement of $\mathrm{F}$ carries along FF (LI). Thus the whole lexical item moves to the spec $\mathrm{CP}$ position. In such a case, if the [+wh] feature of the wh-phrase "which book" is moved to the spec CP position, the movement is not allowed because this movement violates Wh-island constraint in 15). The first wh-word with [+wh] feature constitutes an island for the movement of the feature F of the wh-phrase "which book". The movement of the lexical item the wh-phrase "which book" illustrated in 16) violates 15) as this movement crosses over the wh-word "who". The wh-word "who" formulates a wh-island for the pied-piping of the lexical item the wh-phrase "which book", when the wh-feature F of the wh-phrase "which book" is moved to spec CP position. The pied-piping of the lexical wh-phrase "which book" violates the wh-island constraint, and thus the derivation crashes, and the sentence in 16) is ungrammatical. The representation is illustrated in 17).

15) Wh-island Constraint 
The feature of a wh-phrase cannot be extracted out of the wh-island.

16) ${ }^{*}$ Which book did who read?

17) ${ }^{\star}\left[{ }_{\mathrm{CP}}[+ \text { which book }]_{\mathrm{i}}\right.$ did [IP who read $\mathrm{t}_{\mathrm{i}}$ ?] $]$

Example 5) is the English counterpart of 3). In 5) the specifier [+wh] feature of the head C is weak; the formal feature FF (F) is not carried along or pied-piped. Different from 3), the structure of 5) can be represented as in 18), and the tree diagram of 5) is 19) in which only the feature F moves, while the lexical wh-phrase of "na ben shu" does not piped-pipe with the [+wh] feature of the wh-phrase "na ben shu". As it is shown in 19), the wh-phrase "na ben shu" does not move to the sentence initial position of spec $\mathrm{CP}$ and remains in situ.

18) $\left[{ }_{\mathrm{CP}}[+\mathrm{wh}]\right.$ [IP $_{\mathrm{IP}}$ du le na ben shu?]]

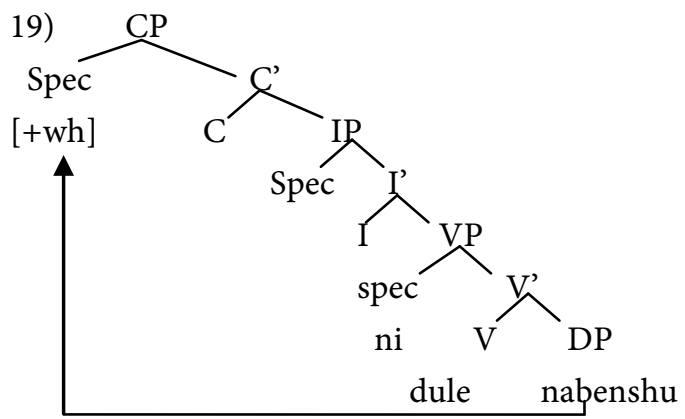

Example 20) is the Chinese counterpart of 12).

20) shei du le na ben shu?

Who read Past which cl. book?

"Who read which book?"

According to the theory of Move represented in 7) and 8), in 20) the [+wh] feature of the wh-word "shei" is unchecked and thus this feature enters into a checking relation with a sublabel of $\mathrm{K}$ as a result of the movement operation. The [+wh] feature F of the wh-word "shei" actually enters into a checking relation with the specifier [+wh] feature of the head C in CP position. In the checking process, Move $\mathrm{F}$ in Chinese does not automatically carry along the FF (F) formal feature of the lexical item the wh-word "shei" as the head [+wh] feature of $\mathrm{C}$ is weak. The [+wh] feature F of the wh-word "shei" moves into spec CP to check the specifier [+wh] feature of the head. And only when F the [+wh] feature of the wh-word "shei" moves into spec CP, the specifier [+wh] feature of the head can be checked. The [+wh] feature of $F$ the [+wh] feature of the wh-word "shei" agrees with the specifier [+wh] feature of the head and thus the specifier [+wh] feature of the head is checked. And as the specifier [+wh] feature of the head is uninterpretable, once it is checked it is erased and does not enter into LF. The derivation converges and the sentence is grammatical.

As it is illustrated in the above English and Chinese examples, Move F of the wh-questions in both languages is universal. Move $\mathrm{F}$ also proves to work in other two types of languages as is demonstrated in the following examples.

In Bulgarian type of languages, all the wh-words must move to the spec of $\mathrm{CP}$ position. As it is illustrated in 21) [19], the wh-word in subject position must always occur in front of the wh-word in object position. In Bulgarian, $\mathrm{C}$ in 
wh-question carries an extremely strong [+wh] feature, and this extremely strong feature attracts the wh-features of all the wh-words to move to Spec CP position in the initial position of the sentence for $[+w h]$ feature checking. The wh-features of all the wh-words must be integrated as a wh-feature cluster. As the [+wh] feature in $\mathrm{C}$ is too strong, the morphological features of all the wh-words piped-pipe with the [+wh] feature cluster. The [+wh] feature in $\mathrm{C}$ is in agreement with the [+wh] feature cluster of all the wh-words. As a result, 21) is interpreted as a wh-question.

21) koj kakvo kupuva

Who what bought

"Who bought what?"

In Czech all the wh-words must raise, but different from Multiply Filled Spec Languages, only one wh-word is moved to the Spec CP position in the front of the sentence. After the raised wh-element, no other wh-word should be allowed to move into the Spec CP position, and there must be another syntactic constituent after it as in 22 a). If two wh-words are raised into the front position of Spec CP as it is the case in Bulgarian, the sentence is ungrammatical as revealed in 22 b). In Czech, wh-words can be isolated by another constituent, which shows that in Czech the wh-words cannot be integrated into one operator [2] [6] [7]. In Czech, the wh-feature of $\mathrm{C}$ is strong but not as strong as in Multiply Filled Spec Languages, because the wh-feature of $C$ can only attract the [+wh] feature of one wh-word to move to Spec CP position. If the [+wh] features of the two wh-words form a feature cluster as it is in Bulgarian and the wh-words are attracted by the strong feature of $\mathrm{C}$ to pied-pipe to Spec $\mathrm{CP}$ position, the sentence turns to be ungrammatical as demonstrated in $22 \mathrm{~b}$ ).

22) a) kdo ho kde videl je nejasme.

Who he where see is difficult-to-understand?

"I do not understand who saw him where?"

b) ${ }^{\star}$ kdo kde ho videl je nejasne.

Who where he see is difficult-to-understand?

To summarize, the study of the wh-feature checking of the wh-features in the wh-questions of the four types of natural languages shows that Move F of feature movement is universal in feature checking. In some languages such as in Chinese no pied-piping of the morphological features of the wh-words is needed while in other types of languages pied-piping of the morphological features of the wh-words is required. In Single Filled Spec Languages such as in English, only the feature of one wh-word is moved to the Spec CP position in the front of the wh-questions, and following economy principle, the morphological feature of the wh-word pied-pipe with the wh-feature so that only one wh-word is attracted to move to the initial position of the sentence. In Multiply Filled Spec Languages such as in Bulgarian, all the features of all the wh-words form a feature cluster and are attracted to move to the Spec CP position in the front of the wh-questions, and all the morphological features of all the wh-words also form a 
morphological feature cluster and pied-pipe with the [+wh] feature cluster so that all the wh-words are raised to the front position of the sentences. In Non-Multiply Filled Spec Languages such as in Czech, only the feature of one wh-word is attracted to move to the Spec CP position in the front of the wh-questions, and the morphological feature of the wh-word pied-pipes with the wh-feature so that only one wh-word is attracted to move to the initial position of the sentence. Besides, another wh-word can be adjoined to $\mathrm{C}$ and all the other wh-words must adjoin to IP. From the above analysis a generalization of the universality of Move F can be made.

\section{Biological Relevance}

Language is human specific. Language is beautiful. Language is perfect. Language is part of nature, as nature is perfect. Nature must be very simple for simplicity is beautiful [1]. Nature's drive for beauty and simplicity triggers, we may make a hypothesis, the universality of Move $\mathrm{F}$ [8]. The minimalist universality is "in many respects an optional solution to conditions it must satisfy" [1]. Move F, as a general universality of languages, attributed to UG, the genetic endowment, accords with Principle of Efficient Computation [8].

23) Principle of Efficient Computation

Linguistic computation is efficient and minimal.

The ability to process language is unique only to the mankind [20]. Phylogenetic and Ontogenetic processing differences can be traced at the neural level [20]. Merge builds a "discrete infinity of structured expressions that are interpretable in a definite way by the conceptual-intentional system of thought and action and by a sensory-motor system for externalization" [21]. Merge may be called the "Basic Property" of language [22]. Merge automatically yields the ubiquitous syntactic phenomenon of displacement [22]. Merge as the fundamental mechanism forming linguistic hierarchies is localized in the ventral anterior portion of Brodmann Area 44, which with its strong neural connection to the posterior temporal cortex, provides a neurobiological basis for the unique human faculty of language [20]. BA 44, situated just anterior to premotor cortex (BA6) and on the lateral surface, inferior to BA9, is also known as pars opercularis of the inferior frontal gyrus, which refers to a subdivision of the cytoarchitecturally defined frontal region of cerebral cortex in the human brain. BA 44 comprises Broca's area, a region involved in semantic tasks. Although we are not sure whether Move F is localized in BA 44 or not, displacement is said to be internal merge and thus displacement must be localized in BA 44. Therefore Move F, pure feature movement, partial displacement of only the features, as a semantic scope requirement in languages, must be relevant to BA 44 .

As far as the three factors of language are concerned, Move F, as a universal principle, must be related with the genetic factors rather than epigenetic factors or nonlinguistic specific factors [8]. Move F, is not learnt by children, instead it might be an innate knowledge children may share in their linguistic operation. This innate knowledge should be able to reveal what faculty of language is. 


\section{Conclusions}

To conclude, from the studies of the four types of natural languages in the world, a general principle in the formation of wh-questions is generated. Move $\mathrm{F}$ is proven to be universal in wh-questions of the world languages, which should be due to the genetic factors of language rather than epigenetic or non-language specific factors.

Move $\mathrm{F}$ as a general principle, is clearly related with biological factors, but how Move F reveals the faculty of language should be further elaborated in the future research. And as merge is considered to be the basic property of language, how merge is operated in the wh-questions should be the topic of the future research.

\section{Acknowledgements}

Ma Daoshan would like to extend his sincere thanks to those present at The Second International Biolinguistics Conference held in Tsinghua University, China (2017/12/8-2017/12/10) for the suggestions and comments on the early draft of this thesis. Special thanks go to Zhang Lianwen, Yang Liexiang and $\mathrm{Hu}$ Wei for their enlightening ideas.

\section{References}

[1] Chomsky, N. (2004) Biolinguistics and the Human Capacity. MTA, Budapest.

[2] Ma, D.S. (2000) A Generative Approach to Interrogative Sentences. M.A. Thesis, Shandong University, Jinan.

[3] Ma, D.S. (2001) The Types of Natural Languages from the Perspective of Generative Studies of Wh-Questions. Contemporary Research in Modern Chinese, 3, 23-37.

[4] Ma, D.S. (2008) The Translation of Interrogative Sentences in Laozi and Its Typological Significance. Theoretical Linguistic Studies, 2, 101-108.

[5] Ma, D.S. (2014) A Syntactic Study of the Interrogative Sentences and Its Typological Significance in the Selected Readings from the Works of Mao Tsetung. Journal of Beijing International Studies University, 10, 14-23.

[6] Ma, D.S. (2015) The Syntactic Studies of Interrogative Sentences in the West and Their Deficiencies. World Book Publishing Co. Ltd., Guangzhou.

[7] Ma, D.S. (2016) An Outline of English and Chinese Syntax. World Book Publishing Co. Ltd., Guangzhou.

[8] Ma, D.S. (2017) Biologically Relevant Universality and Move F. The Second International Biolinguistics Conference, 8-10 December 2017, Beijing, Tsinghua University.

[9] Ma, D.S. (2017) An Outline of English and Chinese Linguistics. World Book Publishing Co. Ltd., Guangzhou.

[10] Ma, D.S. (2004) Is the Mood Auxiliary "Ne" a Clause Typing Marker? Yellow Sea Academic Forum, 4, 150-156.

[11] Ma, D.S. (2006) On the Syntactic Proof of the Interrogative Particle "Ne". Journal of Chinese Linguistics, 12, 100-112.

[12] Ma, D.S. (2006) Is the Question Particle "Ne" a Question Operator? Chinese Foreign Language, 2, 43-47. 
[13] Ma, D.S. (2016) The Distribution and Feature Checking of Interrogative Sentences in Tao Te Ching. International Journal of Language and Linguistics, 4, 230-236. https://doi.org/10.11648/j.ijll.20160406.15

[14] Ma, D.S. (2017) Feature Attraction of Wh-Questions in the Bamboo Slips of Tao Te Ching. International Journal of Research in Humanities, Arts and Literature, 5, 39-50.

[15] Ma, D.S. (2017) Wh-Feature Attraction and Affix Particles in Tao Te Ching. Internationl Journal of Research in Social Sciences, 7.

[16] Ma, D.S. (2017) Left Periphery and Feature Checking of Wh-Questions in Tao Te Ching. BEST: International Journal of Humanities, Arts, Medicine and Sciences (BEST: IJHAMS), 5, 133-144.

[17] Ma, D.S. (2017) Feature Checking of Wh-Questions in the Selected Readings from the Works of Mao Tsetung. International Research Journal of Humanities, Language and Literature, 4, 13-32.

[18] Chomsky, N. (1995) The Minimalist Program. MIT Press, Cambridge, Mass.

[19] Ackema, P. and Neeleman, A. (1998) Optimal Questions. Natural Language and Linguistic Theory, 16, 443-490. https://doi.org/10.1023/A:1006020702441

[20] Zccarella, E. and Friederici, A.D. (2017) The Neurobiological Nature of Syntactic Hierarchies. Neuroscience and Behavioral Reviews, 81, 205-212. https://doi.org/10.1016/j.neubiorev.2016.07.038

[21] Chomsky, N. (2016) What Evolved and How It Might Have Happened. In: Fitch, W., Ed., Psychological Bulletin and Review, Vol. 6, 1-11.

[22] Berwick, R.C. and Chomsky, N. (2017) Why Only Us: Recent Questions and Answers. Journal of Neurolinguistics, 43, 166-177.

https://doi.org/10.1016/j.jneuroling.2016.12.002 